\title{
Study of BL Her type pulsating variable stars using publicly available photometric databases
}

\author{
Monika Jurković ${ }^{1}$ and László Szabados ${ }^{2}$ \\ ${ }^{1}$ Astronomical Observatory of Belgrade, Volgina 7, 11060 Belgrade, Serbia \\ email: mojur@aob.rs \\ ${ }^{2}$ Konkoly Observatory of the Hungarian Academy of Sciences \\ H-1525 Budapest, P.O. Box 67., Hungary
}

\begin{abstract}
BL Her type pulsating variable stars are a subtype of Type II Cepheids, pulsating with periods in the range from 1 to 4 days. The General Catalog of Variable Stars lists 71 objects. For each star from this list, we searched for data in the publicly available photometric databases: AAVSO, ASAS, Catalina Sky Survey, INTEGRAL OMC, LINEAR, NSVS, SuperWASP. The analysis was done separately for each dataset. Here we present first results.
\end{abstract}

Keywords. stars: variable: Cepheids

\section{Data and method}

The list of BL Her variables was gathered from the General Catalogue of Variable Stars (GCVS). Time-series data for each star were collected by searching the publicly available databases: AAVSO, ASAS (Pojmański 1997, 2002, 2003; Pojmański \& Maciejewski 2004, 2005; Pojmański et al. 2005), INTEGRAL OMC, Catalina Sky Survey, SuperWASP (Butters et al. 2010), etc. The data were analysed using Period04 (Lenz \& Breger 2005).

\section{First results}

In the case of V553 Sco, V4410 Sgr, AT Tel, V714 Cyg, V742 Cyg and KO Lyr no data were found. In some cases there were not enough data points to perform the Fourier analysis. Here we present results on three stars we have studied. Our work has not been completed yet.

BD Cas - INTEGRAL data: instrumental errors in the light curve (Fig. 1).

NY Her - AAVSO data: SU UMa type dwarf nova (Kato et al. 2013), (Fig. 2).

UW For - ASAS data: an eclipsing binary (Fig. 3).
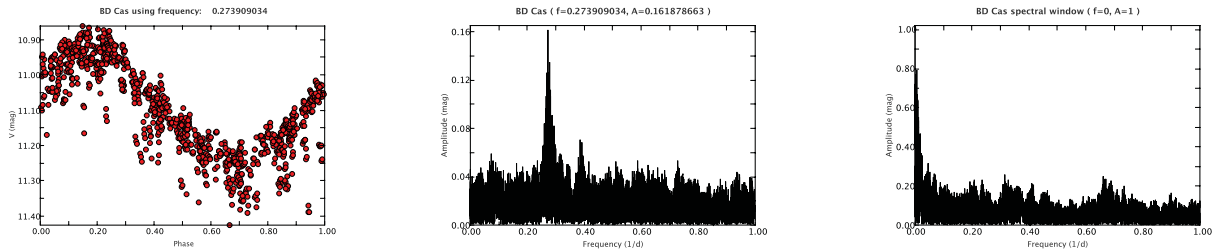

Figure 1. BD Cas from the INTEGRAL data. Left: phase diagram. Middle: Fourier spectrum, Right: spectral window. 

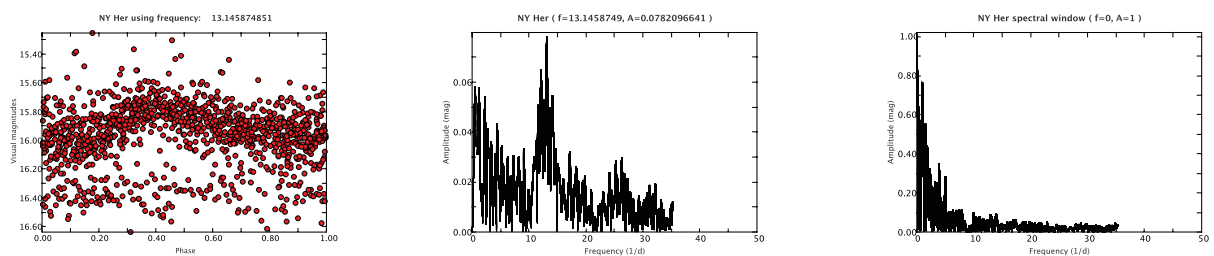

Figure 2. The same as in Fig. 1, but for the AAVSO data of NY Her.
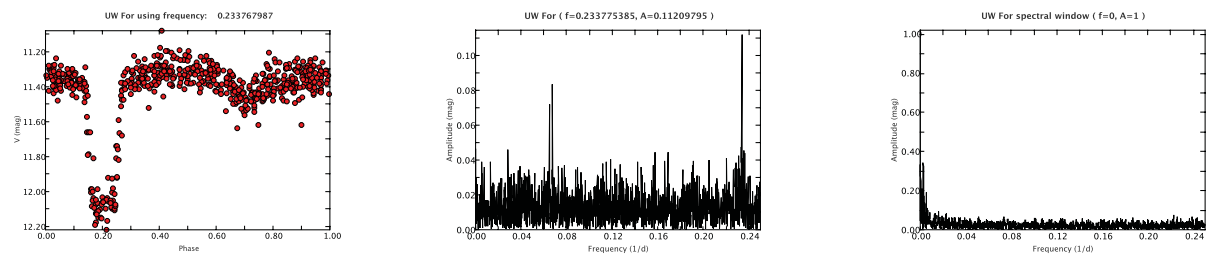

Figure 3. The same as in Fig. 1, but for the ASAS data of UW For.

\section{Conclusion}

As the presented three examples show, the GCVS classification for BL Her stars has to be taken into account with great care. With the completion of our study we hope to make a more reliable classification, and carry out further analysis of all objects.

\section{Acknowledgements}

The authors would like to thank for the IAU travel grant. The authors acknowledge financial support from the Ministry of Education, Science and Technical Development of the Republic of Serbia through project 176004, and the DOMUS 2013 program of the Hungarian Academy of Sciences. This reasearch is based on data from the OMC Archive at CAB (INTA-CSIC), pre-processed by ISDC, the Catalina Sky Survey, and AAVSO. This research has made use of VizieR catalogue access tool, CDS, Strasbourg, France.

\section{References}

Butters, O. W., West, R. G., Anderson, D. R., et al. 2010, A\& A, 510, L10

Kato, T., Hambsch, F-J., Maehara, H., et al. 2013, PASJ, 65, 23

Lenz, P. \& Breger, M. 2005, CoAst, 146, 53

Pojmański, G. 1997, AcA, 47, 467

Pojmański, G. 2002, AcA, 52, 397

Pojmański, G. 2003, AcA, 53, 341

Pojmański, G. \& Maciejewski, G. 2004, AcA, 54, 153

Pojmański, G. \& Maciejewski, G. 2005, AcA, 55, 97

Pojmański, G., Pilecki, B., \& Szczygieł, D. 2005, AcA, 55, 275 